\title{
MODIFIED EXTENDER BASED FORCING
}

\author{
DIMA SINAPOVA AND SPENCER UNGER
}

\begin{abstract}
We analyze the modified extender based forcing from Assaf Sharon's PhD thesis. We show there is a bad scale in the extension and therefore weak square fails. We also present two metatheorems which give a rough characterization of when a diagonal Prikry-type forcing forces the failure of weak square.
\end{abstract}

\section{INTRODUCTION}

In the 1980's Woodin asked if the failure of the Singular Cardinals Hypothesis $(\mathrm{SCH})$ was compatible with the failure of weak square at a singular cardinal $\mu$. The case when $\mu=\aleph_{\omega}$ is of particular interest and remains open. In [5] Gitik and Magidor developed a diagonal extender based forcing construction to violate SCH. This forcing was in part motivated by searching for a way to obtain failure of weak square at a large singular cardinal together with not SCH. (Their forcing has since been refined by Gitik, see for example Section 2 of [4].) However, in his PhD Thesis [8] Sharon showed that this forcing actually adds a weak square sequence.

A positive answer to Woodin's question for a large singular cardinal was obtained by Gitik and Sharon [6]. In fact they obtained the stronger result that the approachability property fails. Cummings and Foreman [1] were able to show that there is a PCF theoretic object called a bad scale in Gitik and Sharon's model, which is responsible for the failure of the approachability property. Gitik and Sharon's forcing admits the addition of collapses in a natural way to make the singular cardinal into $\aleph_{\omega^{2}}$, which gives a positive answer to Woodin's question for $\aleph_{\omega^{2}}$. Recently the authors [9] added collapses to the Gitik and Sharon forcing to make $\kappa$ into $\aleph_{\omega}$. The result was a model where $\mathrm{SCH}$ fails at $\aleph_{\omega}$, but weak square holds. The argument that weak square holds is a modification of Sharon's argument that weak square holds in Gitik and Magidor's model mentioned above. In a second paper [10], we were able to show that the forcing from our previous paper gives the failure of the intermediate weak square principles $\square_{\aleph_{\omega}, \aleph_{n}}$ for $n<\omega$. This is currently the best known partial result towards a positive answer to Woodin's question.

This paper is motivated by a search for other posets which might give a positive answer to Woodin's question at $\aleph_{\omega}$. It is reasonable to ask whether

The first author is partially supported by the National Science Foundation under Grant No. DMS - 1362485. 
there is some way to modify Gitik and Magidor's extender based forcing in order to obtain the failure of weak square. Further if there is such a forcing, then is it possible to add collapses to obtain a positive answer to Woodin's question at $\aleph_{\omega}$. In this paper we show that the answer to the first question is yes and the answer to the second question is no. In particular, we present a version of extender based Prikry forcing from Sharon's $\mathrm{PhD}$ thesis, which we call modified extender based forcing and show that it forces the failure of SCH and the existence of a bad scale, which implies the failure of weak square. We give a strong answer to the second question by proving two metatheorems which characterize when a diagonal Prikry forcing forces the failure of weak square. As an application we show that a reasonable attempt to add collapses to the modified extender based forcing to make $\kappa$ into $\aleph_{\omega}$ will add weak square. This suggests that extender based forcing is unlikely to provide a positive answer to Woodin's question for $\aleph_{\omega}$.

The sections of the paper are organized as follows. In Section 2, we give the definition of the modified extender based forcing and prove some of its properties. The definitions and theorems of this section are all due to Sharon. In Section 3, we show that assuming $\kappa$ is supercompact, there is a bad scale in the extension by the modified extender based forcing. In Section 4, we prove our metatheorems and show that certain extender based forcings with short extenders add weak square.

\section{Modified EXTENDER BASED FORCing}

In order to motivate the definition of the modified extender based forcing, we describe a natural failed attempt to modify the extender based forcing with long extenders from Section 2 of Gitik's chapter in the Handbook of Set Theory [4]. This long extender forcing from [4] is a simplification of the forcing construction of Gitik and Magidor mentioned above. The reader is advised to have a good understanding of it before attempting to read this section of the paper.

As a background assumption, we have an increasing sequence of cardinals $\left\langle\kappa_{n} \mid n<\omega\right\rangle$ with $\sup _{n<\omega} \kappa_{n}=\kappa_{\omega}$ such that each $\kappa_{n}$ carries a $\left(\kappa_{n}, \kappa_{\omega}^{++}\right)$extender $E_{n}=\left\langle E_{n \alpha} \mid \alpha<\kappa_{\omega}^{++}\right\rangle$. Recall that we say $\alpha \leq_{E_{n}} \beta$ if and only if there is a function $f: \kappa \rightarrow \kappa$ such that $j_{n}(f)(\beta)=\alpha$ where $j_{n}$ is the ultrapower map generated by $E_{n}$. For such $\alpha$ and $\beta$ we fix a map $\pi_{\beta \alpha}: \kappa \rightarrow \kappa$ that witnesses $\alpha \leq_{E_{n}} \beta$. We also set $\mu=\kappa_{\omega}^{+}$.

The long extender based forcing is a way of gluing together of $\omega$ many cells. Each cell $\mathbb{Q}_{n}$ has two parts $\mathbb{Q}_{n 0}$ and $\mathbb{Q}_{n 1} \cdot \mathbb{Q}_{n 1}$ is a version of Cohen forcing and $\mathbb{Q}_{n 0}$ is a constrained version of Cohen forcing where some coordinates are controlled by a measure one set from some measure in the extender $E_{n}$. The order on a cell is the order on each of the pieces together with a method for passing from $\mathbb{Q}_{n 0}$ to $\mathbb{Q}_{n 1}$ by selecting an element from the current measure one set and using the constraint to add information to the Cohen part of the condition. 
For Gitik and Magidor the size of the Cohen parts is at most $\kappa_{\omega}$ and a careful read of Sharon's thesis reveals that these large Cohen parts are responsible for the addition of weak square. Unfortunately, the size of the Cohen parts also allows for an easy proof of the Prikry lemma, since the Cohen parts are closed enough to become universal.

A natural attempt to avoid adding weak square is to decrease the size of the Cohen parts in each cell. To this end we define $\mathbb{Q}_{n 1}=\left\{f: \kappa_{\omega}^{++} \rightarrow \kappa_{n} \mid\right.$ $\left.|f| \leq \kappa_{n}\right\}$ and $\mathbb{Q}_{n 0}$ to be as in Definition 2.6 of [4], but with the modified version of $\mathbb{Q}_{n 1}$.

Definition 1. Conditions in $\mathbb{Q}_{n 0}$ are triples $\langle a, A, f\rangle$ where

(1) $f \in \mathbb{Q}_{n 1}$,

(2) $a \subseteq \kappa_{\omega}^{++}$such that $|a|<\kappa_{n}$ and it has a maximal ordinal $\operatorname{mc}(a)$ in the sense of $\leq_{E_{n}}$ which is also its largest element,

(3) $A \in E_{n \mathrm{mc}(a)}$,

(4) if $\beta<\alpha$ with $\alpha, \beta \in a$, then for all $\nu \in A, \pi_{\mathrm{mc}(a) \beta}(\nu)<\pi_{\operatorname{mc}(a) \alpha}(\nu)$, and

(5) if $\alpha, \beta, \gamma \in a$ with $\gamma \leq_{E_{n}} \beta \leq_{E_{n}} \alpha$, then for all $\nu \in \pi_{\operatorname{mc}(a) \alpha}$ " $A$, $\pi_{\alpha \gamma}(\nu)=\pi_{\beta \gamma}\left(\pi_{\alpha \beta}(\nu)\right)$.

We define $(a, A, f) \leq(b, B, g)$ if and only if $f \supseteq g, a \supseteq b$ and $\pi_{\mathrm{mc}(a) \operatorname{mc}(b)}$ " $A \subseteq$ $B$.

The combination of $\mathbb{Q}_{n 1}$ and $\mathbb{Q}_{n 0}$ to form $\mathbb{Q}_{n}$ is the standard one.

Definition 2. Let $\mathbb{Q}_{n}=\mathbb{Q}_{n 0} \cup \mathbb{Q}_{n 1}$ where we set $p \leq q$ if

(1) $p, q \in \mathbb{Q}_{n i}$ for $i \in 2$ and $p \leq q$ or

(2) $p \in \mathbb{Q}_{n 1}, q \in \mathbb{Q}_{n 0}$ and $p \supseteq q \frown \nu$ (defined below) for some $\nu$.

For $\langle a, A, f\rangle \in \mathbb{Q}_{n 0}$ and $\nu \in A$, let $\left.\langle a, A, f\rangle\right\rangle^{-}=f \cup\left\{\left\langle\beta, \pi_{\operatorname{mc}(a), \beta}(\nu)\right\rangle \mid \beta \in\right.$ $a\}$.

We start with some easy propositions.

Proposition 3. (1) $\mathbb{Q}_{n}$ is $\kappa_{n}^{+}$-closed.

(2) $\mathbb{Q}_{n}$ has the $\kappa_{n}^{++}$chain condition.

(3) $\mathbb{Q}_{n 0}$ is $\kappa_{n}$-closed, preserves $\kappa_{n}^{++}$, and collapses $\left(\kappa_{n}^{+}\right)^{V}$ to $\kappa_{n}$.

One can then define a poset $\hat{\mathbb{P}}$ as in Definitions 2.13 and 2.14 of [4]. More precisely, conditions in $\hat{\mathbb{P}}$ are of the form $p=\left\langle p_{n} \mid n<\omega\right\rangle$, with length $\operatorname{lh}(p)$, such that for $n<\operatorname{lh}(p), p_{n} \in \mathbb{Q}_{n 1}$ and for $n \geq \operatorname{lh}(p), p_{n} \in \mathbb{Q}_{n 0}$. The order is given by $q \leq p$ if for all $n, q_{n} \leq_{\mathbb{Q}_{n}} p_{n}$, and also $q \leq^{*} p$ if $q \leq p$ and they have the same length. The naive expectation is that this poset will satisfy the Prikry property (and hence preserve cardinals up to $\kappa_{\omega}$ ) and also force the failure of weak square. Unfortunately, it does not satisfy the Prikry property, in fact it collapses $\kappa_{\omega}$ to be countable. The definition of modified extender based forcing can be seen as a way to fix $\hat{\mathbb{P}}$ in order to recover the Prikry property. 
Let us work for the moment with a condition $p \in \hat{\mathbb{P}}$ and a statement $\varphi$ in the forcing language for $\hat{\mathbb{P}}$. Possible extensions of $p$ are determined by finite sequences of points from the measure one sets in $p$. Let $\vec{\nu}$ be such a sequence, and let $p^{\wedge} \vec{\nu}$ denote the weakest possible extension with respect to $\vec{\nu}$ (below we give a precise definition). If there is a condition $q \leq^{*} p^{\curvearrowleft} \vec{\nu}$ which decides $\varphi$, then we record this condition as $q_{\vec{\nu}}$. To prove the Prikry lemma we would like a direct extension of $p$ which captures each $q_{\vec{\nu}}$. Unfortunately the Cohen parts $f$ are no longer closed enough to do this, since we have reduced their size.

What we are left with is for each $n<\omega$ a function from $\{\vec{\nu} \mid \vec{\nu}$ is a possible extension of $p$ with $\left.\operatorname{lh}\left(p^{\frown} \vec{\nu}\right)>n\right\}$ to $\mathbb{Q}_{n 1}$ given by $\vec{\nu} \mapsto q_{\vec{\nu}}(n)$. Since we cannot capture such a function by a direct extension in $\hat{\mathbb{P}}$, we replace the current $f$-parts with functions of this form under a suitable ordering. We note that if $n$ is larger than the length of $p$, then our $n^{\text {th }}$ function will not depend on $\vec{\nu} \uparrow(n-\operatorname{lh}(p))$.

Let $\left[\prod_{i \geq l} A_{i}\right]^{<\omega}$ denote the set of all sequences $\left\langle\nu_{l}, \ldots, \nu_{n}\right\rangle$, where each $\nu_{i} \in A_{i}$.

Definition 4. Conditions in $\mathbb{P}$ are of the form

$$
p=\left\langle f_{0}, \ldots, f_{l-1},\left\langle a_{l}, A_{l}, f_{l}\right\rangle,\left\langle a_{l+1}, A_{l+1}, f_{l+1}\right\rangle, \ldots\right\rangle
$$

where:

(1) For $n<l, f_{n}:\left[\prod_{i \geq l} A_{i}\right]^{<\omega} \rightarrow \mathbb{Q}_{n 1}$.

(2) For $n \geq l, \operatorname{dom}\left(f_{n}\right)=\left[\prod_{i>n} A_{i}\right]^{<\omega}$, and for each $\vec{\nu} \in \operatorname{dom}\left(f_{n}\right)$, $\left\langle a_{n}, A_{n}, f_{n}(\vec{\nu})\right\rangle \in \mathbb{Q}_{n 0}$.

(3) For $l \leq n<m$, we have $a_{n} \subset a_{m}$.

For $p$ as above and $\nu \in A_{l}, p \frown \nu$ is the condition $\left\langle f_{0}^{\prime}, \ldots, f_{l-1}^{\prime}, f_{l}^{\prime},\left\langle a_{l+1}^{\prime}, A_{l+1}^{\prime}, f_{l+1}^{\prime}\right\rangle, \ldots\right\rangle$, where:

(1) for $n<l=\operatorname{lh}(p)$, for every $h \in \operatorname{dom}\left(f_{n}^{\prime}\right), f_{n}^{\prime}(h)=f_{n}(\nu \frown h)$,

(2) for every $h \in \operatorname{dom}\left(f_{l}^{\prime}\right), f_{l}^{\prime}(h)=\left\langle a_{l}, A_{l}, f_{l}(h)\right\rangle \frown \nu$,

(3) for $n>l,\left\langle a_{n}^{\prime}, A_{n}^{\prime}, f_{n}^{\prime}\right\rangle=\left\langle a_{n}, A_{n}, f_{n}\right\rangle$.

Similarly, define $p \frown \vec{\nu}$.

For $p$ as above with $\operatorname{lh}(p)=l$, we denote $p:=\left\langle p_{n} \mid n<\omega\right\rangle$ or $p=$ $\left\langle f_{0}^{p}, \ldots, f_{l-1}^{p},\left\langle a_{l}^{p}, A_{l}^{p}, f_{l}^{p}\right\rangle,\left\langle a_{l+1}^{p}, A_{l+1}^{p}, f_{l+1}^{p}\right\rangle, \ldots\right\rangle$. Recall that for each $n \geq \operatorname{lh}(p)$, $a_{n}^{p}$ has a $\leq_{E_{n}}$-maximal element which we call $\operatorname{mc}\left(a_{n}^{p}\right)$.

Definition 5. $q \leq^{*} p$ if

(1) $\operatorname{lh}(q)=\ln (p)=l$,

(2) for all $n \geq l, a_{n}^{q} \supseteq a_{n}^{p}$ and $\pi_{\operatorname{mc}\left(a_{n}^{q}\right) \operatorname{mc}\left(a_{n}^{p}\right)}$ " $A_{n}^{q} \subseteq A_{n}^{p}$, and

(3) for all $n<\omega$ and all $\vec{\nu} \in\left[\prod_{i>n} A_{i}^{q}\right]^{<\omega}, f_{n}^{q}(\vec{\nu}) \leq f_{n}^{p}(\pi(\vec{\nu}))$ where $\pi$ is the map that for each $i$ applies $\pi_{\mathrm{mc}\left(a_{i}^{q}\right) \operatorname{mc}\left(a_{i}^{p}\right)}$ to the $i^{\text {th }}$ coordinate of $\vec{\nu}$.

Definition 6. Then $q \leq p$ if for some $\vec{\nu}, q \leq^{*} p^{\frown} \vec{\nu}$. 
It is straightforward to show that the ordering is transitive. The following is standard, but for completeness we sketch the proof.

Lemma 7. $\mathbb{P}$ has the Prikry property: for every sentence in the forcing language $\phi$ and condition $p$, there is $q \leq^{*} p$, such that $q$ decides $\phi$.

Proof. Suppose that $p \in \mathbb{P}$ and $\varphi$ is statement in the forcing language. By the discussion preceding the definition of the forcing there is a $p_{0} \leq^{*} p$ such that for all sequences $\vec{\nu}$ if there is $q \leq^{*} p_{0}^{\overparen{\nu}} \vec{v}$ wich decides $\varphi$, then $p_{0} \vec{\nu}$ decides $\varphi$ in the same way. Note that at this point in the construction, we have fixed the $a$-parts of the conditions we consider and hence all measure one sets will come from the measures $E_{m \mathrm{mc}\left(a_{m}^{p_{0}}\right)}$ for $m \geq \operatorname{lh}\left(p_{0}\right)$.

We now reduce the measure one sets as follows. Suppose that $\vec{\nu}$ is a sequence of ordinals. We partition the set of $\rho$ such that $p_{0} \vec{\nu} \supset \rho$ is a condition into three sets. The set of $\rho$ for which $p_{0} \vec{\nu} \rho$ forces $\varphi$, forces $\neg \varphi$ or doesn't decide $\varphi$. Let $A_{\vec{\nu}}$ be the unique set of the three which is measure one for the appropriate measure. Now for each $k, \bigcap\left\{A_{\vec{\nu}} \mid \operatorname{lh}(\vec{\nu})=k\right\}$ is measure one and hence we can strengthen $p_{0}$ to $p_{1}$ by restricting its measure one sets to these intersections.

To finish the proof we claim that there is a direct extension of $p_{1}$ which decides $\varphi$. Otherwise, we take an extension $q$ of minimal length which decides it (without loss of generality assume that it forces $\varphi$ ). By the construction, we can take $q=p_{1}^{-} \vec{\nu} \rho^{*}$ for some $\vec{\nu}$ and $\rho^{*}$. Now by the reduction of measure one sets we know that $A_{\vec{\nu}}$ is the set of $\rho$ for which $p_{1}^{\neg} \vec{\nu} \rho$ forces $\varphi$. It follows that $p_{1} \vec{\nu}$ forces $\varphi$, a contradiction to the minimality of the length of $q$.

A similar argument gives a standard strengthening of the previous lemma.

Lemma 8. For every dense open set $D$ and condition $p$, there is $q \leq^{*} p$ and $n<\omega$, such that all $r \leq q$ of length $n$ are in $D$.

Proposition 9. $\mathbb{P}$ has the following properties:

(1) $\left\langle\mathbb{P}, \leq^{*}\right\rangle$ is $\kappa_{0}$ - directed closed.

(2) $\left\langle\mathbb{P}\left\lceil[n, \omega), \leq^{*}\right\rangle\right.$ is $\kappa_{n}$-closed, where $\mathbb{P}\lceil[n, \omega):=\{p\lceil[n, \omega) \mid p \in \mathbb{P}\}$.

(3) $\mathbb{P}$ does not add bounded subsets of $\kappa_{0}$.

(4) Both $\mathbb{P}$ and $\left\langle\mathbb{P}, \leq^{*}\right\rangle$ preserve $\mu$

Items 1 through 3 are straightforward. The last item is one of the key properties of this forcing which allows us to argue that there is a bad scale in the extension, so we devote particular attention to its proof. Below we prove that certain restrictions of $\mathbb{P}$ have chain condition. This shows that $\left\langle\mathbb{P}, \leq^{*}\right\rangle$ preserves $\mu$. The fact that $\mathbb{P}$ preserves $\mu$ is due to the Prikry property.

For $n>0$ and $p \in \mathbb{P}_{0}:=\{p \in \mathbb{P} \mid \operatorname{lh}(p)=0\}$, let $\pi_{n}(p)=\left\langle p_{0}, p_{1}, \ldots, p_{n-1}\right\rangle$. Set $\mathbb{P}_{0 n}:=\left\{\pi_{n}(p) \mid p \in \mathbb{P}_{0}\right\}$ with the natural induced ordering from $\leq^{*}$.

Proposition 10. For all $n \geq 0, \mathbb{P}_{0 n+1}$ has the $\kappa_{n}^{++}$-c.c. 
Proof. By induction on $n$. Suppose for contradiction that $\left\{\pi_{n+1}\left(p^{\eta}\right) \mid \eta<\right.$ $\left.\kappa_{n}^{++}\right\}$is an antichain in $\mathbb{P}_{0 n+1}$. We may assume that the part above $n$ of each $p^{\eta}$ is the same, i.e. for some $p \in \mathbb{P}$, for all $m>n$ and $\eta<\kappa_{n}^{++}, p_{m}^{\eta}=p_{m}$. For $m>n$, let $\alpha_{m}=\operatorname{mc}\left(a_{m}^{p}\right)$. For $m>n$, set $i_{m}:=j_{E_{n+1, \alpha_{n+1}}} \circ \ldots j_{E_{m, \alpha_{m}}}$.

We will define functions $f_{m}^{\eta}$ for $n<m, \eta<\kappa_{n}^{++}$as follows.

- If $m=n+1$, for $\nu \in A_{n+1}^{p}$, let $f_{n+1}^{\eta}:=\left[\nu \mapsto f_{n}^{p^{\eta}}(\nu)\right]_{E_{n+1, \alpha_{n+1}}}$. Since $\left|f_{n}^{p^{\eta}}(\nu)\right| \leq \kappa_{n}$, which is below the critical point of $E_{n+1, \alpha_{n+1}}$, we have that $\left|f_{n+1}^{\eta}\right| \leq \kappa_{n}$

- If $m=n+2$, for $\nu \in A_{n+1}^{p}$, let,

$-f_{\nu}^{\eta}:=\left[\delta \mapsto f_{n}^{p^{\eta}}(\nu \frown \delta)\right]_{E_{n+2, \alpha_{n+2}}} ;$

$-f_{n+2}^{\eta}:=\left[\nu \mapsto f_{\nu}^{\eta}\right]_{E_{n+1, \alpha_{n+1}}}$.

As before, $\left|f_{n+2}^{\eta}\right| \leq \kappa_{n}$.

- ... continue in a similar fashion for all $m>n$.

Then each $f_{m}^{\eta}$ is a partial function from $i_{m}\left(\mu^{+}\right)$to $\kappa_{n}$ of size less than or equal to $\kappa_{n}$. Now define a partial function $F_{m}^{\eta}: i_{m}\left(\mu^{+}\right) \rightarrow\{Y\} \cup \kappa_{n}$ by setting

$$
F_{m}^{\eta}(\alpha):= \begin{cases}Y & \text { if } \alpha \in i_{m}\left(a_{n}^{p_{\eta}}\right) \\ f_{m}^{\eta}(\alpha) & \text { if } \alpha \in \operatorname{dom}\left(f_{m}^{\eta}\right)\end{cases}
$$

Let $F^{\eta}$ be the function given by $F^{\eta}(m, \alpha)=F_{m}^{\eta}(\alpha)$. This is a function of size less than $\kappa_{n}^{+}$. So, by applying the $\Delta$-system lemma, we get an unbounded $I \subset \kappa_{n}^{++}$, such that $\left\langle F^{\eta} \mid \eta \in I\right\rangle$ forms a $\Delta$ system, and the functions have the same value on the kernel. Note that this implies that for all $\eta, \delta$ in $I$ and for all $n<m, i_{m}\left(a_{n}^{p_{\eta}}\right) \cap \operatorname{dom}\left(f_{m}^{\delta}\right)=\emptyset$.

By the inductive hypothesis, if $n>0, \mathbb{P}_{0 n}$ has the $\kappa_{n-1}^{++}$-c.c. So let $\eta, \delta$ be distinct points in $I$, such that if $n>0, \pi_{n}\left(p^{\eta}\right)$ and $\pi_{n}\left(p^{\delta}\right)$ are compatible. We will construct $p \in \mathbb{P}_{0}$, such that $\pi_{n+1}(p)$ is a common extension of $\pi_{n+1}\left(p^{\eta}\right)$ and $\pi_{n+1}\left(p^{\delta}\right)$.

Let $\xi<\mu^{+}$be above the supremum of the domains of $f_{k}^{p_{\eta}}(\vec{\delta})$ and $f_{k}^{p_{\delta}}(\vec{\delta})$, for $k \leq n, \vec{\delta} \in \operatorname{dom}\left(f_{k}^{p_{\eta}}\right) \cap \operatorname{dom}\left(f_{k}^{p_{\delta}}\right)$. Also, let $r$ be a common extension of $\pi_{n}\left(p_{\eta}\right)$ and $\pi_{n}\left(p_{\delta}\right)$, such that for all $k<n, a_{k}^{r}=a_{k}^{p^{\eta}} \cup a_{k}^{p^{\delta}} \cup c$, where $c \subset \mu^{+} \backslash \xi$.

For $i<n$, set $a_{i}^{p}=a_{i}^{r}, A_{i}^{p}=A_{i}^{r}$. Then let

$$
a_{n}^{p}=a_{n}^{p^{\eta}} \cup a_{n}^{p^{\delta}} \cup \bigcup_{i<n} a_{i}^{p} \cup\{\hat{\xi}\},
$$

where $\hat{\xi}>\xi$. Then, set

$$
A_{n}^{p}=\pi_{\hat{\xi}, \operatorname{mc}\left(a_{n}^{p^{\eta}}\right)}^{-1}\left(A_{n}^{p^{\eta}}\right) \cap \pi_{\hat{\xi}, \operatorname{mc}\left(a_{n}^{p^{\delta}}\right)}^{-1}\left(A_{n}^{p^{\delta}}\right) .
$$

Finally, for all $m>n$, let

$$
f^{m}=f_{m}^{\eta} \cup f_{m}^{\delta}
$$


This is a well-defined function because the values on the kernel of the $\Delta$ system obtained above are the same.

Denote:

- $f^{m}=\left[\nu \mapsto f_{n}^{m}(\nu)\right]_{E_{n+1, \alpha_{n+1}}} ;$

- $f_{n}^{m}(\nu):=\left[\delta \mapsto f_{n}^{m}(\nu)(\delta)\right]_{E_{n+2, \alpha_{n+2}}}$;

- ... and so on until we reach $m$.

We use " $\forall_{E}^{*}$ " to denote "for a measure one set in $E$ ". Then we have that:

$\forall_{E_{n+1, \alpha_{n+1}}^{*}}^{*} \nu_{n+1} \forall_{E_{n+2}, \alpha_{n+2}}^{*} \nu_{n+2} \ldots \forall_{E_{m}, \alpha_{m}}^{*} \nu_{m}$

$(\dagger): f_{n}^{m}\left(\nu_{n+1}\right) \ldots\left(\nu_{m}\right)=$

$f_{n}^{p_{\eta}}\left(\left\langle\nu_{n+1}, \ldots, \nu_{m}\right\rangle\right) \cup f_{n}^{p_{\delta}}\left(\left\langle\nu_{n+1}, \ldots, \nu_{m}\right\rangle\right)$

and

$$
\operatorname{dom}\left(f_{n}^{m}\left(\nu_{n+1}\right) \ldots\left(\nu_{m}\right)\right) \cap a_{n}^{p}=\emptyset .
$$

Then by taking intersections we have measure one sets $A_{n+1}^{m}, A_{n+2}^{m}, \ldots, A_{m}^{m}$, where each $A_{i}^{m} \in E_{i, \alpha_{i}}$, such that for all $\vec{\nu} \in\left[\prod_{n<i \leq m} A_{i}^{m}\right]^{<\omega}$, we have that the above equality holds.

For $i>n$, let

$$
A_{i}^{p}=\bigcap_{i \leq m<\omega} A_{i}^{m} .
$$

For $i \leq n$, let $f_{i}^{p}=f_{i}^{r}\left\lceil\operatorname{dom}\left(f_{i}^{p}\right)\right.$.

For $\vec{\nu}$ in $\left[\prod_{i>n} A_{i}^{p}\right]^{<\omega}$, let

$$
f_{n}^{p}(x)\left(\nu_{n+1}, \ldots, \nu_{m}\right)=f_{n}^{m}(x)\left(\nu_{n+1}\right) \ldots\left(\nu_{m}\right) .
$$

Then $p$ is as desired.

Corollary 11. $\left(\mathbb{P}_{0}, \leq^{*}\right)$ preserves all cardinals in the interval $\left[\kappa_{n}^{++}, \kappa_{n+1}\right]$ for each $n$, and so preserves $\kappa_{\omega}$ and $\mu$.

Proof. (Sketch) Otherwise, suppose that for some $n$ we have that some regular $V$-cardinal $\tau \in\left[\kappa_{n}^{++}, \kappa_{n+1}\right]$ is collapsed. Let $p \in \mathbb{P}_{0}$, and $\lambda<\tau$ be such that $p \Vdash_{\mathbb{P}_{0}} \dot{h}: \lambda \rightarrow \tau$ is onto. Using that $\mathbb{P}_{0 n+1}$ has the $\kappa_{n}^{++}$c.c. and $\left(\mathbb{P}_{0} \uparrow[n+1, \omega), \leq^{*}\right)$ is $\kappa_{n+1}$-closed, we build conditions $\left\langle q_{\alpha} \mid \alpha<\lambda\right\rangle$, such that:

(1) each $q_{\alpha}\lceil n+1=p\lceil n+1$,

(2) $\left\langle q_{\alpha}\left\lceil[n+1, \omega)|\alpha<\kappa\rangle\right.\right.$ is $\leq^{*}$-decreasing,

(3) each $q_{\alpha} \Vdash \dot{h}(\alpha) \in X_{\alpha}$ for some $X_{\alpha} \subset \tau$ in $V$ with $\left|X_{\alpha}\right|<\kappa_{n}^{++}$.

Let $X=\bigcup_{\alpha<\lambda} X_{\alpha}$, and let $q \leq^{*} p$ be such that for all $\alpha<\lambda, q \leq^{*} q_{\alpha}$. Then $q \Vdash_{\mathbb{P}_{0}} \operatorname{ran}(\dot{h}) \subset X$, but $|X|<\tau$. Contradiction.

Theorem 12. (Assaf Sharon, [8]) In $V^{\mathbb{P}}, 2^{\omega}<\kappa_{\omega}, \kappa_{\omega}^{\omega}=\kappa_{\omega}^{++}$, and there are no very good scales at $\kappa_{\omega}$.

In the generic extension, $\kappa_{\omega}$ is no longer strong limit, because $\left(\mathbb{P}, \leq^{*}\right)$ is only $\kappa_{0}$-closed. So there are no bounded subsets added to $\kappa_{0}$ and $2^{\kappa_{0}}$ becomes $\mu^{+}$. 


\section{THE BAD SCALE}

In this section we prove the following theorem.

Theorem 13. If $\kappa_{0}$ is indestructibly supercompact in $V$ then in $V^{\mathbb{P}}$ there is a bad scale of length $\mu$ in $\prod_{n<\omega} \kappa_{n}^{++}$and so weak square at $\kappa_{\omega}$ fails.

We assume that $V$ is prepared so that the supercompactness of $\kappa_{0}$ is preserved by forcing with $\mathbb{P}_{0}$. Fix a scale $\left\langle g_{\alpha} \mid \gamma<\mu\right\rangle \in V$ in $\prod_{n} \kappa_{n}^{++}$of length $\mu$. Set $S:=\left\{\gamma<\mu \mid \omega<\operatorname{cf}(\gamma)<\kappa_{0}, \gamma\right.$ is a bad point for $\left\langle g_{\gamma}\right| \gamma<$ $\mu\rangle$. By standard reflection arguments $S$ is stationary in $V$. Also, since $\mathbb{P}_{0}$ preserves $\mu$ and is $\kappa_{0}$-directed closed, $\left\langle g_{\gamma} \mid \gamma<\mu\right\rangle$ remains a bad scale after forcing with $\mathbb{P}_{0}$. More precisely, if $G_{0}$ is $\mathbb{P}_{0}$-generic, a point of cofinality less than $\kappa_{0}$ is bad in $V$ iff it is bad in $V\left[G_{0}\right]$, and the set $S$ is stationary in $V\left[G_{0}\right]$ (since $\kappa_{0}$ remains supercompact in $V\left[G_{0}\right]$ ).

Definition 14. For $p \in \mathbb{P}$, set $t(p)=p\lceil[\operatorname{lh}(p), \omega)$. Let $\mathbb{D}:=\{t(p) \mid p \in \mathbb{P}\}$, and $t(p) \leq_{\mathbb{D}} t(q)$ if for some large enough $n, 1 \mathcal{\complement}^{-} p\left\lceil[n, \omega) \leq_{\mathbb{P}} \mathcal{\complement}^{\frown} q \uparrow[n, \omega)\right.$.

Note that both $\mathbb{P}$ and $\mathbb{P}_{0}$ project to $\mathbb{D}$

Lemma 15. $\mathbb{P} / \mathbb{D}$ has the $\mu$-chain condition.

Proof. Let $H^{*}$ be $\mathbb{P}_{0}$-generic and let $H$ be the induced $\mathbb{D}$-generic object. It is enough to show that $\mathbb{P} / H$ has the $\mu$-cc in $V\left[H^{*}\right]$. First note that for any $p \in \mathbb{P}$ with $p\left\lceil[n, \omega) \in H\right.$ for some $n$, there are an $n^{*}$ and $p^{\prime} \in H^{*}$ such that $p^{\prime} \leq_{\mathbb{P}_{0}} 1 \frown p \uparrow\left[n^{*}, \omega\right)$.

Let $\left\langle p_{\alpha} \mid \alpha<\mu\right\rangle$ be a sequence of elements of $\mathbb{P} / H$ in $V\left[H^{*}\right]$. By the above note, for all $\alpha$, there is an $n_{\alpha}$, such that $1 \frown p_{\alpha} \uparrow\left[n_{\alpha}, \omega\right) \in H^{*}$. By refining our list of conditions, we can assume that there is $n^{*}$ such that $n_{\alpha}=n^{*}$ for all $\alpha$. By extending each condition we can assume it has length $n^{*}$. Now if we take the first $\kappa_{n^{*}-1}^{++}$conditions, then by Proposition 10 there are two which are compatible.

So if $H$ is $\mathbb{D}$-generic, since $\mathbb{P}_{0}$ projects to $\mathbb{D}$, we have that $S$ is stationary in $V[H]$. Then by the $\mu$-chain condition of $\mathbb{P} / H, S$ is stationary after forcing with $\mathbb{P}$.

Let $G$ be $\mathbb{P}$-generic.

Lemma 16. Let $\tau<\kappa_{0}$ be a regular uncountable cardinal in $V$ (and so in $V[G]$ ), and suppose $V[G] \models A \subset O N$, o.t. $(A)=\tau$. Then there is a $B \in V$ such that $B$ is an unbounded subset of $A$.

Proof. Let $p \in G, p \Vdash \dot{h}: \tau \rightarrow \dot{A}$ enumerate $\dot{A}$. By the Prikry lemma, define $\mathrm{a} \leq^{*}$-decreasing sequence $\left\langle p_{\alpha} \mid \alpha<\tau\right\rangle$, such for every $\alpha<\tau, p_{\alpha} \leq^{*} p$ and there is $n_{\alpha}<\omega$, such that every $q \leq p_{\alpha}$ with length $n_{\alpha}$ decides $\dot{h}(\alpha)$. Then there is an unbounded $I \subset \tau$ and $n<\omega$ such that for all $\alpha \in I, n=n_{\alpha}$. Let $p^{\prime}$ be stronger than all $p_{\alpha}$ for $\alpha<\tau$. By appealing to density, we may assume that $p^{\prime} \in G$. Let $q \leq p^{\prime}$ be a condition in $G$ with length $n$, and set $B=\{\gamma \mid(\exists \alpha \in I) q \Vdash \dot{h}(\alpha)=\gamma\}$. Then $B$ is as desired. 
Lemma 17. Suppose that in $V[G], h \in \prod_{n} \kappa_{n}^{++}$. Then there is a function $\bar{h} \in \prod_{n} \kappa_{n}^{++}$in $V$, such that for all large $n, h(n) \leq \bar{h}(n)$.

Proof. Let $p$ force that $\dot{h}$ is as in the statement of the lemma. For simplicity assume that the length of $p$ is 0 . For all $n$ by the Prikry property there is some $p_{n} \leq^{*} p$ and $k_{n}$, such that every $r \leq p_{n}$ of length $k_{n}$ decides the value of $\dot{h}(n)$. Since there are only $\kappa_{n}^{++}$such possibilities, by shrinking the measure one sets of $p_{n}\left\lceil[n+1, \omega)\right.$, we may assume that $k_{n}=n+1$. In other words, every $n+1$-step extension of $p_{n}$ decides $\dot{h}(n)$.

Doing this inductively, we arrange that $\left\langle p_{n}\right| n\langle\omega\rangle$ are $\leq^{*}$ - decreasing. Let $q$ be a lower bound, of length 0 . For every $n+1$-step extension $q \frown \vec{\nu}$, let $\gamma_{\vec{\nu}}$ be the value of $\dot{h}(n)$ decided by $q \frown \vec{\nu}$. Define $\bar{h}(n)=\sup _{\vec{\nu}:|\vec{\nu}|=n+1} \gamma_{\vec{\nu}}<\kappa_{n}^{++}$

Then $q$ forces $\dot{h}(n) \leq \bar{h}(n)$.

Corollary 18. $\left\langle g_{\beta} \mid \beta<\mu\right\rangle$ is a bad scale in $V[G]$

\section{TWo METATHEOREMS}

In this section we prove two metatheorems which roughly characterize when a diagonal Prikry forcing forces weak square to hold. We start with an abstract definition of what it means for a poset to be a diagonal Prikry forcing.

Definition 19. A poset $\left(\mathbb{P}, \leq, \leq^{*}\right)$ is a diagonal Prikry forcing if there is an increasing sequence of regular cardinals $\left\langle\kappa_{n} \mid n<\omega\right\rangle$ where we define $\mu=\left(\sup _{n<\omega} \kappa_{n}\right)^{+}$such that:

(1) Every $p \in \mathbb{P}$ is of the form $p=\left\langle p_{n} \mid n<\omega\right\rangle$.

(2) There is a function $\ell: \mathbb{P} \rightarrow \omega$ such that $p \leq^{*} q$ if and only if $\ell(p)=\ell(q)$ and $p \leq q$.

(3) If we define $\mathbb{P}^{n}=\{p\lceil[n, \omega) \mid p \in \mathbb{P}, \ell(p)=0\}$ with the induced ordering from $\leq$ and $\mathbb{D}=\bigcup_{n<\omega} \mathbb{P}^{n}$ where we let $p\lceil[n, \omega) \leq q \uparrow$ $[m, \omega)$ if there is $k \geq \max (m, n)$ such that $p\lceil[k, \omega) \leq q \uparrow[k, \omega)$ in $\mathbb{P}^{k}$, then we have the following:

(a) $\mathbb{P}^{n}$ is countably closed and $<\kappa_{n}$-distributive.

(b) For $n<m$, the map taking $p \uparrow[n, \omega)$ to $p \uparrow[m, \omega)$ is a good projection from $\mathbb{P}^{n}$ to $\mathbb{P}^{m}$.

(c) $\mathbb{P}$ induces a generic for $\mathbb{D}$.

(4) Working in $V\left[\mathbb{P}^{0}\right]$, for all cardinals $\delta<\kappa_{0}$ which are successors of singular cardinals of cofinality $\omega$, there is a condition $p \in \mathbb{P} / \mathbb{D}$ such that $p$ forces that " $\delta$ is preserved and for all ordinals $\gamma$ with $\operatorname{cf}(\gamma)=\delta$ and all $A \subseteq \gamma$ unbounded, there is $B \subseteq A$ unbounded with $B$ in $V$."

(5) It is forced by $\mathbb{P}$ that " $\mu$ is preserved and its predecessor $\mu^{-}$has the property that for all $\alpha<\mu^{-}, \alpha^{\omega}<\mu^{-}$.

We note that most posets which we think of as Diagonal Prikry forcing fit this definition. Examples include Diagonal Prikry forcing (see [4]), Gitik 
and Sharon's forcing [6], Gitik and Magidor's extender based forcing [5], the main forcing from this paper and any variation of these posets with collapses added.

We are now ready to prove two metatheorems about diagonal Prikry forcing and weak square. For the first one we will use the following fact, which appears in the last section of [9]. For the benefit of the reader we sketch the main points of the proof, see [9] for the complete argument.

Fact 20. Suppose that $\kappa$ is a singular strong limit, and $\left\langle\tau_{n}\right| n\langle\omega\rangle$ is an increasing sequence of regular cardinals with limit $\kappa$. Then $\prod_{n} \operatorname{Col}\left(\tau_{n}, \kappa^{+}\right) /$finite adds weak square at $\kappa$.

Proof. Denote $\mathbb{C}_{n}:=\operatorname{Col}\left(\tau_{n}, \kappa^{+}\right)$and let $\dot{C}_{n}$ be the $\mathbb{C}_{n}$-name for the generic club of $\kappa^{+}$of order type $\tau_{n}$. Let $H$ be $\prod_{n} \mathbb{C}_{n} /$ finite-generic. We say that a point $\gamma$ is good if there is $\left[c_{n}\right] /$ fin $\in H$ such that for all large $n$, $c_{n} \Vdash_{\mathbb{C}_{n}} \gamma \in \lim \dot{C}_{n}$. Then in $V[H]$, the set $X:=\left\{\gamma<\kappa^{+} \mid \gamma\right.$ is good $\}$ is $>\omega$ -club. Now for every $\gamma \in X$, let $\vec{c}_{\gamma}$ and $n_{\gamma}$ witness that $\gamma \in X$. I.e. for all $n \geq n_{\gamma}, \vec{c}_{\gamma}(n) \Vdash_{\mathbb{C}_{n}} \gamma \in \lim \dot{C}_{n}$. By density we may also assume that each $\vec{c}_{\gamma}(n)$ decides $\dot{C}_{n} \cap \gamma$, and let $Z_{\gamma, n}$ be that value. For $\gamma \in X$, define

$$
\mathcal{C}_{\gamma}:=\left\{Y \subset \gamma \mid Y \text { is a club in } \gamma,\left(\exists k \geq n_{\gamma}\right)\left(Y \subset \bigcap_{n \geq k} Z_{\gamma, n}\right)\right\} .
$$

And for $\gamma \in \operatorname{cof}(\omega) \backslash X$, set $\mathcal{C}_{\gamma}=\{Y\}$, where $Y$ is any cofinal $\omega$ sequence in $\gamma$. Then one can show that $\left\langle\mathcal{C}_{\gamma} \mid \gamma \in X \cup \operatorname{cof}(\omega)\right\rangle$ is a $\square_{\kappa}^{*}$ sequence.

Theorem 21. Suppose that $\mathbb{P}$ is a diagonal Prikry forcing and that there is a model $V_{0}$ with the same cardinals as $V$ where $\mu^{-}$is strong limit, $\mathbb{P}^{0} \in V_{0}$, $V=V_{0}[A]$ for some generic $A$ and $\mathbb{P}^{0}$ is mutually generic with the forcing to add $A$. If for all $n<\omega$ forcing with $\mathbb{P}^{n}$ over $V_{0}$ collapses $\mu$, then forcing with $\mathbb{P}$ over $V$ adds $a \square_{\mu^{-}}^{*}$-sequence.

Proof. By clause (3)(c) of Definition 19 and the conditions of the theorem, it is enough to show that forcing with $\mathbb{D}$ over $V_{0}$ adds a weak square sequence. We can assume that for all large $n,\left\langle\mathbb{P}^{n}, \leq^{*}\right\rangle$ collapses $\mu$ to have cofinality $\kappa_{n}$. Then by the above mentioned fact, it follows that weak square is added by $\mathbb{D}$. We remark that the proof is a essentially a reconstruction of the proof of the following fact.

An application of the above theorem is that the standard way of replacing long extenders with short extenders in the modified EBF will add weak square. In this application we take $A$ to be trivial. We remark that forcing with short extenders is the usual method of interleaving collapses in extender based forcing.

The natural way of combining short extenders with modified EBF is to replace the $a \subseteq \kappa_{\omega}^{++}$in the definition of $\mathbb{Q}_{n 0}$ with an order preserving function $a: \kappa_{\omega}^{++} \rightarrow \kappa_{n}^{+n+2}$ with extra properties. Without further modification 
this poset will not have the $\kappa_{\omega}^{++}$-cc. To recover the $\kappa_{\omega}^{++}$chain condition, we have to define an equivalence relation and take a quotient of the poset. We refer the reader to [3] or [7] for details of this method. We let $\mathbb{P}_{s}$ be this modified short extender based forcing and we will show that it adds weak square by applying Theorem 21 .

We isolate a relevant part of $\mathbb{P}_{s}$ given by the following definition.

Definition 22. Let $\mathbb{Q}_{n 0}^{*}$ be the poset whose conditions are of the form $\langle a, A, f\rangle$ where $a$ is a function as in the definition of the short extender forcing (see [2] Definition 2.1 and the modification Definition 2.11), $A \in$ $E_{n \max (\operatorname{ran}(a))}$ and $f$ is as in clause (2) of Definition 4 and the ordering is the natural one combining $\leq *$ from the short extender forcing and the modified EBF.

It is easy to see that $\mathbb{Q}_{n 0}^{*}$ adds an order preserving map from $\kappa_{\omega}^{++}$to $\kappa_{n}^{+n+2}$. Unfortunately, it is not true in this case that $\mathbb{D}$ is $\prod_{n<\omega} \mathbb{Q}_{n 0}^{*} /$ fin. The issue is that the final order we use on $\mathbb{P}_{s}$ involves the equivalence relation required to regain the $\kappa_{\omega}^{++}$-cc. To this end we will show that even if we quotient $\mathbb{Q}_{n 0}^{*}$ by the restriction of the equivalence relation on $\mathbb{P}_{s}$, then it still collapses $\mu$. This will be enough to apply Theorem 21 , since we can take $\mathbb{D}$ to be the $\bmod$ finite product of the restricted $\mathbb{Q}_{n 0}^{*}$. For definiteness we denote this restricted poset $\left(\mathbb{Q}_{n 0}^{*}, \rightarrow\right)$ and for those familiar with this type of forcing we note that we are using the equivalence relation $\leftrightarrow_{n, 3}$ (See for example Definition 22 of [7]).

Before we prove that this poset collapses $\mu$ we prove an easy claim that seems to be required in the proof.

Claim 23. Let $f$ be a part of some condition in $\mathbb{Q}_{n 0}^{*}$. For all $m>n$, the set $\left\{\alpha<\kappa_{\omega}^{++} \mid \exists A_{n+1} \ldots \times A_{m} \forall \vec{\nu} \in \vec{A}, \alpha \in \operatorname{dom}(f(\vec{\nu}))\right\}$ has size at most $\kappa_{n}$.

Proof. If the set had size greater than $\kappa_{n}$, then using the completeness of the associated measures we would have a single sequence of measure one sets witnessing membership for more than $\kappa_{n}$ different $\alpha$ 's. This is impossible, since then there will be a $\vec{\nu}$ such that $|\operatorname{dom}(f(\vec{\nu}))|>\kappa_{n}$.

Claim 24. $\left(\mathbb{Q}_{n 0}^{*}, \rightarrow\right)$ collapses $\mu$

Proof. Let $R \subseteq \mu^{+} \cap \operatorname{cf}(\omega)$ be such that $|R|=\kappa_{n}^{+}$and for every $\gamma \in R$, there is an unbounded set $R_{\gamma} \subseteq \gamma$ of order type $\kappa_{\omega}$. For every $c \in \gamma^{\omega}$ let the equivalence class of $c$ be the set of $\left\{d \in \gamma^{\omega} \mid c, d\right.$ coincide on a tail end \} . The number of these equivalence classes is $\mu$, so we enumerate them as $\left\langle c_{\eta}^{\gamma} \mid \eta<\mu\right\rangle$.

Let $H$ be generic for $\left(\mathbb{Q}_{n 0}^{*}, \rightarrow\right)$. In $V[H]$ define $\phi: R \rightarrow \mu$ by setting $\phi(\gamma)=\eta$ if there are $p \in H$ and $c \in \gamma^{\omega}$ such that $c \in c_{\eta}^{\gamma}, c \subseteq \operatorname{dom}\left(a^{p}\right)$ and $a^{p}(c)$ coincides on a tail end with the least $\omega$ sequence of 2 -good ordinals in its supremum. Here we take the least in some canonical well-ordering fixed in advance.

First we show that $\phi$ is well-defined. Suppose that for some $\gamma$ we have conditions $p, q \in H$ and $c, c^{\prime}$ in $\gamma^{\omega}$ such that 
- $c \subseteq \operatorname{dom}\left(a^{p}\right), c^{\prime} \subseteq \operatorname{dom}\left(a^{q}\right)$

- $a^{p}(c)$ and $a^{q}\left(c^{\prime}\right)$ both coincide on a tail end with the least $\omega$ sequence in their respective supremum.

Let $r \in H$ be a common extension of $p$ and $q$. Since $a^{r}$ is order preserving, we define $\bar{\gamma}=\sup \left(a^{r}(c)\right)=\sup \left(a^{r}\left(c^{\prime}\right)\right)$. Using the definition of $\leftrightarrow_{n, 3}$, we have that $\operatorname{ran}\left(a^{r}\right)$ contains a tail end of the least $\omega$ sequence of 2-good ordinals in $\bar{\gamma}$ and each of $c$ and $c^{\prime}$ must map to this $\omega$-sequence. So $c$ and $c^{\prime}$ coincide on a tail.

Next we show that the range of $\phi$ is unbounded in $\mu$. For every $\delta<\mu$, we claim that the set $D=\left\{p \mid(\exists \gamma \in R)(\exists \eta>\delta)\left(\exists c \in c_{\eta}^{\gamma}\right)\right.$ such that $p$ and $c$ witness $\phi(\gamma)=\eta\}$ is dense. Given a condition $q=\langle a, A, f\rangle$, let $d=\operatorname{dom}(a) \cup\left\{\alpha<\kappa_{\omega}^{++} \mid \exists A_{n+1} \ldots \times A_{m} \forall \vec{\nu} \in \vec{A}, \alpha \in \operatorname{dom}(f(\vec{\nu}))\right\}$. By Claim 23 this has size at most $\kappa_{n}$. Now let $\gamma \in R$ be such that $\left|R_{\gamma} \backslash \beta\right|=\kappa_{\omega}$, where $\beta:=\sup (d \cap R)$. Denote $R_{\gamma}^{\prime}:=R_{\gamma} \backslash \beta$. $\left(R_{\gamma}^{\prime}\right)^{\omega}$ has size $\mu$, and so there is some $\eta, \delta<\eta<\mu$, such that $c_{\eta}^{\gamma} \subset R_{\gamma}^{\prime}$. Construct an extension $\left\langle a^{\prime}, A^{\prime}, f^{\prime}\right\rangle$, such that $c_{\eta}^{\gamma} \subset \operatorname{dom}\left(a^{\prime}\right)$, and $a^{\prime}\left(c_{\eta}^{\gamma}\right)=b$, where $\min (b)>\sup (\operatorname{ran}(a))$, and $b$ coincides on a tail end with the least $\omega$-sequence of 2 -good ordinals cofinal in $\sup (b)$. We also shrink the measure one sets $A_{i}^{q}$ to ensure that for all relevant $\vec{\nu}, \operatorname{dom}\left(f^{\prime}(\vec{\nu})\right) \cap \operatorname{dom}\left(a^{\prime}\right)=\emptyset$.

It follows that $\phi$ collapses $\mu$

So by Theorem 21 we get:

Corollary 25. $\mathbb{P}_{s}$ adds a weak square sequence.

It follows that the natural attempt to bring the construction form the previous two sections to $\aleph_{\omega}$ will add weak square, since such a forcing will induce a generic for $\mathbb{P}_{s}$.

The inclusion of $A$ in Theorem 21 allows us to apply the theorem to situations like the one in the authors' previous paper [9] where $\mu^{-}$is first forced not to be strong limit and then diagonal Prikry forcing is defined afterward.

We now prove the second of our two metatheorems.

Theorem 26. Let $\mathbb{P}$ be a diagonal Prikry forcing. If for some $n$, $\Vdash_{\mathbb{P} n}$ " $\mu$ is preserved, $\mathbb{P} / \mathbb{D}$ is $\mu$-cc and there is a $\mu$-supercompactness embedding with critical point $\kappa_{0} . "$, then it is forced by $\mathbb{P}$ that $\square_{\mu^{-}}^{*}$ fails.

Proof. Let $H^{*}$ be $\mathbb{P}^{n}$-generic over $V$ and let $H$ be the induced $\mathbb{D}$-generic. By hypothesis $\mathbb{P} / H$ is $\mu$-cc and there is $j: V\left[H^{*}\right] \rightarrow M$ witnessing that $\kappa_{0}$ is $\mu$-supercompact.

We assume it is forced by $\mathbb{P} / H$ over $V[H]$ that there is a weak square sequence $\left\langle\mathcal{C}_{\alpha} \mid \alpha<\mu\right\rangle$. We may assume that for all $\alpha<\mu$ there is $\dot{D}_{\alpha} \in \mathcal{C}_{\alpha}$ such that ot $\left(\dot{D}_{\alpha}\right)=\operatorname{cf}(\alpha)$ and for all $C \in \mathcal{C}_{\alpha}$, ot $(C)<\mu^{-}$.

Work in $M$ with the forcing $j(\mathbb{P} / H)$. Let $\gamma=\sup j$ " $\mu$. By clause (4) of Definition 19 there is a $p^{*} \in j(\mathbb{P} / H)$ such that $p^{*}$ forces $\mu$ is preserved and for all unbounded subsets $A \subseteq \gamma$ there is a $B \subseteq A$ unbounded with $B \in M$. 
So $p^{*}$ forces that there is $\dot{E}_{\gamma} \in j(\overrightarrow{\mathcal{C}})_{\gamma}$ of ordertype $\mu$ and extending $p^{*}$ if necessary we can assume that there is a club $E \subseteq \gamma$ in $M$ which $p^{*}$ forces to be a subset of $E_{\gamma}$.

Let $D=\{\alpha<\mu \mid j(\alpha) \in E\}$. By standard arguments $D$ is $<\kappa_{0}$-club. Let $\zeta$ be such that $D \cap \zeta$ has ordertype $\mu^{-}$.

Claim 27. For all $x \subseteq D \cap \zeta$ with ot $(x)=\omega$, there is $p_{x} \in \mathbb{P} / H$ such that $p_{x}$ forces $x \subseteq C$ for some $C \in \mathcal{C}_{\zeta}$.

The claim is easy by noticing that $j(x)=j$ " $x \subseteq E \cap j(\zeta)$ which $p^{*}$ forces is a subset of $E_{\gamma} \cap j(\zeta) \in j(\overrightarrow{\mathcal{C}})_{j(\zeta)}$. Using the fact that $\mathbb{P} / H$ is $\mu$-cc, we can find a generic $G$ for $\mathbb{P} / H$ which contains $p_{x}$ for $\mu$ many $x$. This is a contradiction as there are only $\mu^{-}$many possibilities for the $C$ of the claim and each has ordertype less than $\mu^{-}$so by clause (5) of Definition 19 there can be at most $\mu^{-}$such subsets.

We note that the assumption $\Vdash_{\mathbb{P}^{n}} \mathbb{P} / \mathbb{D}$ is $\mu$-cc is typical for diagonal Prikry forcings. The modified extender based forcing from this paper will satisfy it by the proof of Lemma 15.

Theorems 21 and 26 provide a dichotomy for approaching solutions to Woodin's question, "Is it consistent that both $\mathrm{SCH}$ and weak square fail at $\aleph_{\omega}$ ?". Suppose that one constructs a diagonal Prikry forcing as above, which forces the failure of SCH at $\aleph_{\omega}$. The above two theorems taken together say roughly that weak square holds in the extension if and only if each $\mathbb{P}^{n}$ collapses $\mu$.

\section{REFERENCES}

1. James Cummings and Matthew Foreman, Diagonal Prikry extensions, Journal of Symbolic Logic 75 (2010), no. 4, 1383.

2. Moti Gitik, Blowing up the power of a singular cardinal, Annals of Pure and Applied Logic 80 (1996), no. 1, $17-33$.

3. _ Blowing up power of a singular cardinalwider gaps, Annals of Pure and Applied Logic 116 (2002), no. 1-3, 1 - 38.

4. , Prikry-type forcings, Handbook of Set Theory (Matthew Foreman and Akihiro Kanamori, eds.), Springer Netherlands, 2010, pp. 1351-1447 (English).

5. Moti Gitik and Menachem Magidor, Extender based forcings, The Journal of Symbolic Logic 59 (1994), 445-460.

6. Moti Gitik and Assaf Sharon, $\mathrm{On} \mathrm{SCH}$ and the approachability property, Proceedings of the American Mathematical Society (2008), 311-320.

7. Moti Gitik and Spencer Unger, Adding $\omega$-sequences to a singular cardinal.

8. Assaf Sharon, Weak squares, scales, stationary reflection and the failure of sch, Ph.D. Thesis, Tel Aviv University (2005).

9. Dima Sinapova and Spencer Unger, Combinatorics at $\aleph_{\omega}$, Annals of Pure and Applied Logic 165 (2014), no. 4, 996 - 1007.

10. Scales at $\aleph_{\omega},(2014)$, Accepted for publication in the Israel Journal of Mathematics. 